\title{
APF foam does reduce caries in primary teeth
}

\author{
What is the effect of bi-annual professional application of acidulated \\ phosphate fluoride foam on caries increment in primary teeth?
}

\author{
Jiang H, Bian Z, Tai BJ, Du MQ, Peng B. The effect of a bi-annual \\ professional application of APF foam on dental caries increment \\ in primary teeth: 24-month clinical trial. J Dent Res 2005; 84: \\ 265-268
}

Design A double-blind, cluster randomised, placebo-controlled trial was conducted.

Intervention Acidulated phosphate fluoride foam (APF; $1.23 \%$ ) or placebo (fluoride-free) foam were applied by a dental practitioner twice per year for 2 years. Oral examinations were undertaken at baseline and after 2 years, following World Health Organization criteria for caries diagnosis.

Outcome measure Caries increment was evaluated after 2 years. Results In total, 392 children of age 3-4 years were recruited, of whom 209 were randomised to the test group and 183 to the control. One hundred and sixty-seven test and 151 control children completed the study. There was no mean caries increment (measured as decayed, missing or filled primary teeth; $\mathrm{dmfs}$ ) in $38.3 \%$ of test and $26.5 \%$ of control group children. The figure for dmfs was 3.8 in the test and 5.0 in the control group ( $P$ 0.03). A significant difference was also observed in caries increment on approximal surfaces between test and control groups $(\mathrm{P}<0.01)$.

Conclusions Bi-annual professional application of APF foam, over 2 years, was effective in reducing caries increment in primary teeth.

\section{Commentary}

There cannot be many dentists who graduated in the UK in the 1970s and early 1980s who do not remember practising applying APF gel in foam trays to their fellow students, and most of those will still remember the sharp, saliva-inducing flavours of the gels, and the struggle to keep the trays, and their dignity, in place for the required 4-min application period. This paper reports on a well-designed, and carefully conducted randomised controlled clinical trial of a modern development of that gel, APF foam.

There were always concerns regarding the potential fluoride toxicity to children of ingesting excess gel (which contains around 10fold more fluoride by weight than an adult toothpaste), and it was to address this issue that APF foam was developed. This product has the same fluoride concentration (1.23\% or $12300 \mathrm{ppm})$ and $\mathrm{pH}(\mathrm{pH}$ $3-4)$, as APF gels, but requires only about one-fifth the quantity, by weight, to adequately cover a dental arch. This study demonstrated that a bi-annual APF foam application in 3-4 year old nursery school children reduced their dmfs increment by $24 \%$ after a period of
2 years (four applications of APF foam), with the reduction mainly affecting approximal, rather than occlusal, surfaces.

This study adds weight to the evidence supporting the value of preventive interventions involving fluoride administration in children of nursery-school age, especially those located in moderate to high caries-prevalence locales. ${ }^{1}$ The protocol for the application of the APF foam here, however, involved the use of foam trays, which sometimes required trimming in order to fit a particular child. Once trimmed, the trays were filled with the APF foam, and the children required to keep biting into the trays for 4 min while leaning forwards, so that saliva and excess foam could drain extra-orally onto a plastic plate. In contrast, fluoride varnish is quick and easy to apply. Its bland flavour means that it is well-tolerated by children as young as 1 year of age, ${ }^{2}$ and there is evidence that it has a similar efficacy to APF foam as a caries-preventive intervention. ${ }^{2,3}$ Since APF foam and fluoride varnish are comparable in efficacy, it would seem that ease of application would make fluoride varnish the caries-preventive intervention of choice from the perspective of both the healthcare worker and the child.

It is interesting that only around $10 \%$ of the children in this study were having their teeth brushed at least twice a day at home. Brushing twice a day with a pea-sized amount (a smear if $<2$ years of age) of a $1000 \mathrm{ppm}$ fluoride paste, and spitting out rather than rinsing on completion of brushing, remains one of the most effective preventive interventions we have. ${ }^{1}$ All caries-preventing interventions involving fluoride toothpaste, gels, foams or varnishes in nursery-school-age children, especially those located in medium to high caries prevalence areas, are to be welcomed. It must remain a priority, though, to encourage all parents to accept and act upon their responsibility to brush their own children's teeth twice a day until the children are old enough to do it for themselves (probably not until at least the age of 7 years).

\section{Dafydd Evans}

Dental School, University of Dundee, Dundee, Scotland, UK

1. Prevention and Management of Dental Decay in the Pre-school Child. SIGN 83. Edinburgh: Scottish Intercollegiate Guideline Network; 2005.

2. Weintraub JA, Ramos-Gomez F, Jue B, et al. Fluoride varnish efficacy in preventing early childhood caries. J Dent Res 2006; 85:172-176.

3. Marinho VC, Higgins JP, Logan S, Sheiham A. Topical fluoride (toothpastes, mouthrinses, gels or varnishes) for preventing dental caries in children and adolescents (Cochrane Review). Cochrane Library. Chichester: John Wiley; 2003 , issue 4

Evidence-Based Dentistry (2007) 8, 7. doi:10.1038/sj.ebd.6400461 